\title{
ELASTICITY WITH MIXED FINITE ELEMENT
}

\author{
OUADIE KOUBAITI ${ }^{1}$, JAOUAD EL-MEKKAOUI ${ }^{2}$, \\ AND AHMED ELKHALFI ${ }^{3}$ \\ ${ }^{1,3}$ Departement of Genie Mecanique \\ Sidi Mohammed ben abdellah University \\ Faculte des Sciences et Techniques \\ B.P. 2202-Route d'Imouzzer - Fez, MOROCCO \\ ${ }^{2}$ Faculty Polydisciplinaire \\ University of Sultan Moulay Slimane \\ Mghila BP: 592 Beni Mellal, MOROCCO
}

\begin{abstract}
In this article we are intending to solve the Navier-Lame problem in 2D with Dirichlet and Neumann boundaries, using the mixed finite element $P^{1}$-bubble $P^{1}$. We want to introduce a new weak formulation of this problem with help of another new unknown which is equal to divergence of the displacement. We do the necessary calculations of this problem in order to come up with a Matlab program that visualizes the numerical solution.Some numerical results which are shown, prove that our method is more efficient than the ordinary finite element.
\end{abstract}

AMS Subject Classification: 74S05, 78M10, 80M10

Key Words: elasticity, mixed finite element, Matlab

Received: June 19, 2017; Accepted: August 16, 2018;

Published: September 10, 2018 doi: 10.12732/caa.v22i4.1

Dynamic Publishers, Inc., Acad. Publishers, Ltd. http://www.acadsol.eu/caa

\section{INTRODUCTION}

Since 2002, the article [1] entitled by Matlab Implementation of the finite element method in elasticity, thanks to the authors of this work J. Alberty, 
Kiel, C.Carstensen, Vienna, SA Funken, Kiel and R. Klose, that have a great contribution in computing the numerical solution that is the approximation of the exact solution which is the unknown in the Navier-Lame equation, using the ordinary finite element methods programmed by Matlab. In fact, nobody has thought to apply the mixed finite element Method to the equation NavierLame, that will be the subject of our research.

This study is based on the calculation of the numerical solution using the mixed finite element method P1-bubble P1 and to do this we have to create another new unknown by setting $\psi$ equal to the divergence of the displacement, geting a couple of unknown $(u, \psi)$. Comparing the numerical results found in the article cited above mentioned in article [1], we will prove that the new method is more accurate and effecient.

\section{MODEL EQUATION}

We propose the numerical Method employs the mixed finite element (P1bubble,P1) to calculate the numerical solution of the displacement, and its discrete divergence,to the following 2D Navier -Lam problem.Let's consider $\Omega \subset$ $\mathbb{R}^{2}$ be a bounded Lipschitz domain with polygonal boundary $\Gamma=\Gamma_{N} \cup \Gamma_{D}$, with $\Gamma_{D}$ is a closed subset of the boundary with positive length, which called Dirichlet conditions; however $\Gamma_{N}$ is theNeumannboundary condition.The 2 components of the displacements can not satisfy either Dirichlet- or Neumann conditions.Given $f \in L^{2}(\Omega), M \in L^{\infty}\left(\Gamma_{D}\right)^{2 \times 2}, h \in H^{1}\left(\Gamma_{D}\right)$ and $g \in L^{2}\left(\Gamma_{N}\right)$ as well as the positive parameters $\lambda$ and $\mu$. The equation below is governed as follows:

Solid object is deformed under the action of forces applied.A point in the solid,originally in $(x, y)$, after sometime it will come into $(X, Y)$, the vector $u=\left(u_{1}, u_{2}\right)=(X-x, Y-y)$ is called displacement. When the movement is small and the solid is elastic,then HOOK's law gives a relationship between the stress tensor and the strain tensor.

$\sigma=\lambda \operatorname{tr}(\varepsilon) I_{2}+2 \mu \varepsilon$ is the stress tensor; $\varepsilon=\frac{1}{2}\left(\nabla u+(\nabla U)^{T}\right)$ is the strain tensor; $I_{2}$ is the identity matrix; $\mu$ is the shear modulus (or rigidity), where $\lambda$ is Lam's first parameter.

Navier's equation is given by the law of conservation moment $\rho a=\operatorname{div} \sigma$ with a is the acceleration and $\rho$ is the density of material. On the other hand, 
$\operatorname{div} \sigma=\lambda \operatorname{div}\left(\operatorname{tr}(\varepsilon) I_{2}\right)+2 \mu \operatorname{div} \varepsilon$.

Then we have

$$
\operatorname{div} \sigma=\lambda \operatorname{div}\left(\operatorname{tr}(\varepsilon) I_{2}\right)+\mu \operatorname{div}(\operatorname{grad} u)+\mu \operatorname{div}(\operatorname{grad} u)^{T} .
$$

With a simple calculation, we find that

$$
\operatorname{div}\left(\operatorname{tr}(\varepsilon) I_{2}\right)=\operatorname{div}(\operatorname{grad} u)^{T}=\operatorname{grad}(\operatorname{div}(u)) .
$$

Then we get

$$
\rho a=\mu \Delta u+(\lambda+\mu) \operatorname{grad}(\operatorname{div} u) .
$$

If the solid is in dynamic equilibrium then we have $\rho a+f=0, \mathrm{f}$ are the external forces applied to the solid.Finally,we find out the equation $f=$ $-\mu \Delta u-(\lambda+\mu) \operatorname{grad}(\operatorname{div} u)$.

We create a new unknown $\psi=\nabla . u$ that is equal to divergence of the displacement. The equation of Navier-Lame become :

$$
\left\{\begin{aligned}
-\mu \Delta u-(\lambda+\mu) \nabla \psi=f & \text { in } \Omega, \\
\psi=\nabla \cdot u & \text { in } \Omega, \\
M . u=h & \text { on } \Gamma_{D}, \\
\mu \frac{\partial u}{\partial n}+\lambda \nabla \cdot u n=g & \text { on } \Gamma_{N} .
\end{aligned}\right.
$$

We need to introduce the following functional spaces

$$
\begin{aligned}
& h^{1}(\Omega)=\left\{u: \Omega \rightarrow \mathbb{R} \backslash u, \frac{\partial u}{\partial x}, \frac{\partial u}{\partial y} \in L^{2}(\Omega)\right\}, \\
& V_{0}(\Omega)=\left\{u \in H^{1}(\Omega)=\left[h^{1}(\Omega)\right]^{2} \backslash M . u=0 \text { on } \Gamma_{D}\right\}, \\
& V(\Omega)=\left\{u \in H^{1}(\Omega)=\left[h^{1}(\Omega)\right]^{2} \backslash M . u=h \text { on } \Gamma_{D}\right\}, \\
& M(\Omega)=L_{0}^{2}(\Omega)=\left\{q \in L^{2}(\Omega) \backslash \int_{\Omega} q=0\right\} .
\end{aligned}
$$

The variational formulation of the Navier-Lame problem (2.1) is as follows Find $(u, \psi) \in V(\Omega) \times M(\Omega)$ such that

$$
\left\{\begin{aligned}
& a(u, v)+b_{N}(v, \psi)=L(v) \forall v \in V_{0}(\Omega), \\
& b(u, q)-d(\psi, q)=0 \quad \forall q \in M(\Omega),
\end{aligned}\right.
$$


and bilinear forms

$$
\left\{\begin{aligned}
a(u, v) & =\int_{\Omega} \mu \nabla u: \nabla v d \Omega, \\
b(v, q) & =\int_{\Omega}(\lambda+\mu) q \nabla . v d \Omega, \\
b_{N}(v, q) & =b(v, q)-\int_{\Gamma_{N}} \mu q n . v d \Gamma_{N}, \\
d(\psi, q) & =\int_{\Omega}(\lambda+\mu) \psi q d \Omega, \\
L(v) & =\int_{\Omega} f \cdot v d \Omega+\int_{\Gamma_{N}} g \cdot v d \Gamma_{N} .
\end{aligned}\right.
$$

The existence and uniqueness of the solution $(u ; \psi)$ of the system $(2.2)$ and its equivalence with (2.1) are well established by the verification the continuity and coercivity for the bilinear forms and the continuity of linear form $L$, defined above in (2.3) and the Theorem 3.1 in [3].

\section{FINITE ELEMENT DISCRETISATION}

To apply the method of mixed finite element P1-bubble/P1 for the variational problem (2.2).We need some mathematical tools, then we us the approximation of the standard Galerkin method,for more explication we can see in the articles $[6,7,8,9,10]$.Let's consider a uniform triangulation $T_{h}$ of the rectangular domain $\Omega$,whereh $>0$ is the maximum diameter of all elements $K \in T_{h}$, and $T_{h}$ consists of triangles in two dimensions. We assume that we have a sequence of triangulations $\left(T_{h}\right)_{h} \quad h \rightarrow 0$.

Let $\lambda_{1}^{K}, \lambda_{2}^{K}, \lambda_{2}^{K}$ be the barycentric coordinates with respect to a triangle K. $\mu^{K}$ is the bubble function associated with the triangle $K$ defined by $\mu^{K}=$ $\lambda_{1}^{K} \lambda_{2}^{K} \lambda_{3}^{K}$ in $\mathrm{K}$ and equal to 0 elsewhere. We define the discrete domain and two approached spaces as follow:

$$
\Omega_{h}=\bigcup_{k=1}^{n} T_{k},
$$

$\Omega_{h}$ is closed and if $\Omega$ is polygon then $\Omega_{h}=\bar{\Omega}$ and $\Gamma_{h}=\partial \Omega_{h}=\partial \Omega=\Gamma$ 


$$
\begin{aligned}
& V_{h}=\left\{\left.\varphi_{h} \in H^{1}\left(\Omega_{h}\right) \backslash \varphi_{h}\right|_{K}=\sum_{i=1}^{3} \alpha_{i}^{K} \lambda_{i}^{K}+\beta^{K} \mu^{K}(x) \alpha_{i}^{K},\right. \\
& \left.\beta^{K} \in \mathbb{R},\left.M \cdot \varphi_{h}\right|_{\Gamma_{D}} \cap K=0, \forall K \in \mathrm{T}_{h}\right\}, \\
& M_{h}=\left\{\left.\psi_{h} \in L_{0}^{1}\left(\Omega_{h}\right) \backslash \psi_{h}\right|_{K}=\sum_{i=1}^{3} \theta_{i}^{K} \lambda_{i}^{K}, \theta_{i}^{K} \in \mathbb{R}, \forall K \in \mathrm{T}_{h}\right\} \subset \mathbb{P}_{1}(K) .
\end{aligned}
$$

$\mathbb{P}_{1}(K)$ is the space of polynomials defined on the triangle $K$ of the degree lower or equal to 1.

The functions of $V_{h} \times M_{h}$ are not globally affine in all $\Omega$,but only affine by piece.

On the other hand,they are generally continuous.the functions of the space are completely determined by their values in each of the mesh vertices. For the solution of an elasticity problem,the displacement/div-displacement $(u / \psi)$ finite element discretizations are effective in [5]. Let $V_{h}$ be the finite element displacement interpolation space and $M_{h}$ be the finite element div-displacement interpolation space (corresponding to the spaces $V(\Omega)$ and $M=L_{0}^{2}(\Omega)$ of the continuous problem.

The functions of the space $V_{h}$ are completely determined by their values in each of the mesh vertices.Moreover the dimension of the space $V_{h}$ is $N-n_{s}$, with $\mathrm{N}$ is the overall number of vertices and $n_{s}$ the number of vertices on the boundaries. Then the finite element problem is:

Let's seek $u_{h} \in V_{h}$ considering $M . u_{h}=h_{h}$ on $\Gamma_{D}$ and $\psi_{h} \in M_{h}$ and

$$
\left\{\begin{array}{r}
a_{h}\left(u_{h}, v_{h}\right)+b_{N h}\left(v_{h}, \chi_{h}\right)=L_{h}\left(v_{h}\right) \forall v_{h} \in V_{h} \\
b_{h}\left(u_{h}, q_{h}\right)-d_{h}\left(\psi_{h}, q_{h}\right)=0 \forall q_{h} \in M_{h}
\end{array}\right.
$$

where

$$
\left\{\begin{array}{r}
a_{h}\left(u_{h}, v_{h}\right)=\int_{K} \mu \nabla u_{h}: \nabla v_{h} d K \\
b_{h}\left(v_{h}, q_{h}\right)=\int_{K}(\lambda+\mu) q_{h} \nabla \cdot v_{h} d K \\
b_{N h}\left(v_{h}, q_{h}\right)=b_{h}\left(v_{h}, q_{h}\right)-\int_{\Gamma_{N, h}} \mu q_{h} n_{K} \cdot v_{h} d \Gamma_{h} \\
d_{h}\left(\psi_{h}, q_{h}\right)=\int_{K}(\lambda+\mu) \psi_{h} q_{h} d K \\
L_{h}\left(v_{h}\right)=\int_{K} f \cdot v_{h} d K+\int_{\Gamma_{N, h}} g \cdot v_{h} d \Gamma_{N, h}
\end{array}\right.
$$


With $\Gamma_{N, h}=\Gamma_{N} \bigcap \partial K$ and $n_{K}$ the normal on K.

The existence and uniqueness of the solution of the mixed formulation (3.1)is shown by using.

The continuity of the bilinear forms a on $V_{h} \times V_{h}, b_{N}$ on $V_{h} \times M_{h}$, b on $V_{h} \times M_{h}$ and d on $M_{h} \times M_{h}$ is clear by using the korn's inequality. On the other hand the coercivity of the bilinear form a on $V_{h}$ and d on $M_{h}$ is hold by using theirs coercivity on $V(\Omega)$ and $M(\Omega)$ respectively since $V_{h} \subset V(\Omega)$.We can see the uniform inf - sup condition uniformly of the bilinear form $b$ in [4] with respect to the mesh-size.

It can be proved that uniform inf - sup condition for the bilinear form $b_{N h}$ on $V_{0 h} \times M_{0 h}$ it means we have to prove the existence of a constant $\vartheta^{\prime}>0$ independent of the mesh-size as the following theorem clarifies

Theorem 3.1. There exist $\vartheta>0$ such as

$$
\forall q_{h} \in M_{h}, \exists u_{h} \in V_{h}, u_{h} \neq 0 b_{\Gamma}\left(u_{h}, q_{h}\right) \geq \vartheta\left\|u_{h}\right\|_{1, \Omega}\left\|q_{h}\right\|_{0, \Omega} .
$$

This theorem guarantee the verification of the condition inf - sup of the bilinear form $b_{\Gamma}$. It should be noted that

$$
b_{\Gamma}\left(u_{h}, q_{h}\right)=b\left(u_{h}, q_{h}\right)-\int_{\Gamma_{N, h}} \mu q_{h} n_{K} \cdot v_{h} d \Gamma_{h}, \forall u_{h} \in V_{h} .
$$

Proof. First we prove that the bilinear form b verifies (3.3) of Theorem 3.1.

It is assumed that the triangulation $\mathrm{T}_{h}$ is uniformly regular.Let $q_{h} \in M_{h}$ be fixed $M_{h} \subset M$ and that the bilinear form $b$ satisfies the inf - sup's condition in $V \times M$ so that there exist $u \in V$ and $b\left(u, q_{h}\right) \geq \beta\|u\|_{1, \Omega}\left\|q_{h}\right\|_{0, \Omega}$.with $\beta>0$ independent of $q_{h}$, but $\mathrm{u}$ depends of $q_{h}$.for this $\mathrm{u}$ we have just show that $u_{h} \in V_{h}$ clarified below :

$$
b\left(u_{h}, q_{h}\right)=b\left(u, q_{h}\right), \quad\left\|u_{h}\right\|_{1, \Omega} \leq c\|u\|_{1, \Omega}
$$

The relations (3.4) are the object of the later lemma (3.2).where $c>0$ is independent of $q_{h}$ and $\mathrm{h}$.

Indeed if (3.4) are checked so

$$
b\left(u_{h}, q_{h}\right)=b\left(u, q_{h}\right) \geq \beta\|u\|_{1, \Omega}\left\|q_{h}\right\|_{0, \Omega}
$$

$\geq \frac{\beta}{c}\left\|u_{h}\right\|_{1, \Omega}\left\|q_{h}\right\|_{0, \Omega}$, just take $\beta^{\prime}=\frac{\beta}{c}$

We have $\int_{\Gamma} \mu q n . v d \Gamma \leq c^{\prime}|\mu|\|v\|_{1, \Omega}\|q\|_{0, \Omega}$ then, $V_{h} \subset V$ and $M_{h} \subset M$ 
we obtain

$\int_{\Gamma} \mu q_{h} n . v_{h} d \Gamma \leq c^{\prime}|\mu|\left\|v_{h}\right\|_{1, \Omega}\left\|q_{h}\right\|_{0, \Omega}$, then we have $b_{N}\left(v_{h}, q_{h}\right) \geq\left(\beta^{\prime}-\right.$ $\left.c^{\prime}\right) \mu\left\|q_{h}\right\|_{0, \Omega}\left\|v_{h}\right\|_{1, \Omega}$. Also we get $\vartheta=\beta^{\prime}-c^{\prime} \mu$ when we have $\mu<\frac{\beta^{\prime}}{c^{\prime}}$

Lemma 3.2. there exist $u_{h} \in V_{h}$ and $c>0$ so that $u$ is fixed and $\forall q_{h} \in M_{h}$

$$
b\left(u_{h}, q_{h}\right)=b\left(u, q_{h}\right),\left\|u_{h}\right\|_{1, \Omega} \leq c\|u\|_{1, \Omega}
$$

Proof. First we define the linear operator $R_{h} \in \mathrm{L}\left(\mathrm{V}, \mathrm{V}_{\mathrm{h}}\right)$, which verifies $\forall v_{h} \in$ $V_{h} \exists ! v \in V$, so that $R_{h} v=v_{h}$. This operator is a projector of $V$ on $V_{h}$ it is well defined.Indeed Lax Milgramm ensures the unique existence of the variational problem :find $v$ in $\mathrm{V}$ so as

$$
\int_{\Omega} \nabla\left(R_{h} v-v\right) \cdot \nabla v_{h} d x=0, \forall v_{h} \in V_{h}
$$

If we take $v_{h}=R_{h} v$ in (3.6) we obtain $\left\|\nabla R_{h} v\right\|_{0, \Omega}^{2}=\int_{\Omega} \nabla v . \nabla v_{h} d x$ then $\left\|\nabla R_{h} v\right\|_{0, \Omega}^{2} \leq\left\|\nabla R_{h} v\right\|_{0, \Omega}\|\nabla v\|_{0, \Omega}$ we conclude that $\left\|\nabla R_{h} v\right\|_{0, \Omega} \leq \|$ $\nabla v \|_{0, \Omega}$ we find that $R_{h}$ is continuous the sense of $H_{0}^{1}(\Omega) \cap V$, and by the

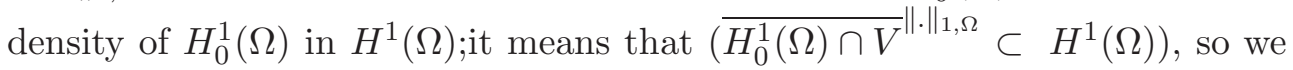
have its continuity in the space $V$ then,we suppose that the operator $R_{h}$ checks the next properties of estimation, that is to say : there exist $C>0$ independent of $\mathrm{h}$ and $v$

$$
\left\|\left(R_{h} v-v\right)\right\|_{0, \Omega} \leq c h\|\nabla v\|_{0, \Omega}
$$

The above findings are workable for all $v \in V$.Now, we can prove that the first relation of (3.5) in lemma (3.2), as follow:

$$
\int_{\Omega_{h}} \nabla \cdot u_{h} q_{h} d x=\int_{\Omega_{h}} \nabla \cdot u q_{h} d x
$$

by applying the Green formula, we found $\int_{\Omega_{h}} \nabla q_{h} \cdot u_{h} d x=\int_{\Omega_{h}} \nabla q_{h} \cdot u d x$ but $\nabla q_{h}$ is constant when $q_{h} \in \mathbb{P}_{1}(K)$. So, $\int_{K} u_{h} d x=\int_{K} u d x \forall K$ by the definition of the space $V_{h}$,for every function $u_{h}$ of $V_{h}$ is determined by the relation $u_{h}=$ $\sum_{i=1}^{3} u_{h}\left(a_{i}^{K}\right) \lambda_{i}(x)+\beta^{K} \mu^{K}(x) \forall x \in K$, we have

$$
\begin{aligned}
\int_{K} u_{h} d x & =\sum_{i=1}^{3} u_{h}\left(a_{i}^{K}\right) \int_{K} \lambda_{i}(x) d x+\beta^{K} \int_{K} \mu^{K}(x) d x \\
& =\sum_{i=1}^{3} R_{h} u\left(a_{i}^{K}\right) \int_{K} \lambda_{i}(x) d x+\beta^{K} \int_{K} \mu^{K}(x) d x
\end{aligned}
$$




$$
=\int_{K} R_{h} u d x
$$

Finally,we just to take $u_{h}=R_{h} u$ who verifies $b\left(u_{h}, q_{h}\right)=b\left(u, q_{h}\right)$.

Now let prove the second relation of lemma (3.2) we have

$$
u_{\left.h\right|_{K}}=\sum_{i=1}^{3} R_{h} u\left(a_{i}^{K}\right) \lambda_{i}+\beta^{K} \mu^{K},
$$

with a simple writing $u_{\left.h\right|_{K}}=R_{h} u+\beta^{K} \mu^{K}$ and $\left\|u_{h}\right\|_{1, \Omega}^{2}=\sum_{K \in \mathbf{T}_{\mathbf{h}}}\left\|u_{h}\right\|_{1, K}^{2}$, then

$$
\begin{aligned}
\left\|u_{h}\right\|_{1, \Omega}^{2} & \leq \sum_{K \in \mathbf{T}_{\mathbf{h}}}\left(\left\|R_{h} u\right\|_{1, K}+\left|\beta^{K}\right|\left\|\mu^{K}\right\|_{1, K}\right)^{2} \\
& \leq 2 \sum_{K \in \mathbf{T}_{\mathbf{h}}}\left(\left\|R_{h} u\right\|_{1, K}^{2}+\left|\beta^{K}\right|^{2}\left\|\mu^{K}\right\|_{1, K}^{2}\right) \\
& \leq 2\left\|R_{h} u\right\|_{1, \Omega}^{2}+2 \sum_{K \in \mathbf{T}_{\mathbf{h}}}\left|\beta^{K}\right|^{2}\left\|\mu^{K}\right\|_{1, K}^{2}
\end{aligned}
$$

By the continuity of the operator $R_{h}\left\|u_{h}\right\|_{1, \Omega}^{2} \leq c\|u\|_{1, \Omega}^{2}+2 \sum_{K \in \mathbf{T}_{\mathbf{h}}}$ $\left.\beta^{K}\right|^{2}\left\|\mu^{K}\right\|_{1, K}^{2}$ By using the relation $\int_{K} u d x=\int_{K} u_{h} d x=\int_{K} R_{h} u d x+$ $\beta^{K} \int_{K} \mu^{K} d x, \beta^{K}=\frac{\int_{K}\left(u-R_{h} u\right) d x}{\int_{K} \mu^{K} d x}, \int_{k} \mu^{K} d x=\frac{|K|}{60}$, and using Cauchy-Schwarz it gives, the existence of $c>0$ independent of $\mathrm{h}$ and $\mathrm{K}$ so as $\left|\beta^{K}\right|^{2} \leq c \frac{\left\|u-R_{h} u\right\|_{0, K}^{2}}{h^{2}}$ the function $\mu^{K}$ is bounded in the sense of the norm : $\|,\|_{1, K}$, and by the relation (3.7) we get $\left\|u_{h}\right\|_{1, \Omega} \leq c\|u\|_{1, \Omega}$

Finally, it was also shown that the element: $\mathbb{P}_{1}-$ bubble $\backslash \mathbb{P}_{1}$ satisfies the condition inf - sup discreet

\section{ALGEBRIC PROBLEM}

In this section we introduce the Matrices $\mathrm{A}, B_{N}, \mathrm{~B}, \mathrm{D}, \mathrm{L}$ related to the bilinear forms $a_{h}, b_{N h}, b_{h}, d_{h}, L_{h}$ respectively in the following way and we can express 
the bilinear forms according to the operators as well defined here

$$
\left\{\begin{array}{r}
a_{h}\left(u_{h}, v_{h}\right)=\left(A u_{h}, v_{h}\right) \forall\left(u_{h}, v_{h}\right) \in V_{h}(\Omega) \times V_{h}(\Omega) \\
b_{N h}\left(v_{h}, q_{h}\right)=\left(B_{N} v_{h}, q_{h}\right) \forall\left(q_{h}, v_{h}\right) \in M_{h}(\Omega) \in \times V_{h}(\Omega) \\
b_{h}\left(u_{h}, q_{h}\right)=\left(B v_{h}, q_{h}\right) \forall q_{h} \in M_{h}(\Omega) \in \times V_{h}(\Omega) \\
d_{h}\left(\psi_{h}, q_{h}\right)=\left(D \psi_{h}, q_{h}\right) \forall\left(\psi_{h}, q_{h}\right) \in M_{h}(\Omega) \times M_{h}(\Omega) \\
L_{h}\left(v_{h}\right)=L v_{h} \forall v_{h} \in V_{h}(\Omega)
\end{array}\right.
$$

With(4.1), we find that the discrete formulation (3.1) can be expressed as a system of operator equations

$$
\left\{\begin{array}{r}
A u_{h}+B_{N}^{t} \psi_{h}=L \\
B u_{h}-D \psi_{h}=0
\end{array}\right.
$$

We find that the discrete formulation can be expressed as a system of linear equations as well

$$
\left(\begin{array}{cc}
A & B_{N}^{t} \\
B & -D
\end{array}\right)\left(\begin{array}{l}
u_{h} \\
\psi_{h}
\end{array}\right)=\left(\begin{array}{l}
L \\
0
\end{array}\right)
$$

with $u_{h}=\left(u_{x}, u_{y}\right)^{t}$, we can express the algebric system (4.2) as follows

$$
\left(\begin{array}{ccc}
A & 0 & B_{N, x}^{t} \\
0 & A & B_{N, y}^{t} \\
B_{x} & B_{y} & -D
\end{array}\right)\left(\begin{array}{c}
u_{x} \\
u_{y} \\
\psi
\end{array}\right)=\left(\begin{array}{c}
L_{x} \\
L_{y} \\
0
\end{array}\right)
$$

Let $\left\{\varphi_{1} ; \varphi_{2} \ldots ; \varphi_{n}\right\}$ be the finite element basis formed of scalar functions $\varphi_{i}, i=1$...n.In practice the two components of $\left(u_{h}^{x}, u_{h}^{y}\right)$ of $u_{h}$ are always appreciated by one space finite element.Let $\mathrm{N}$ be the number of nodes in the finite element mesh, and $n=N-n_{s}$ with ns the number of vertices on the boundaries.The basis of the space $V_{h} B_{V_{h}}=\left\{\phi_{1}=\left(\varphi_{1}, 0\right) \ldots \phi_{n}=\left(\varphi_{n}, 0\right), \phi_{n+1}=\right.$ $\left.\left(0, \varphi_{1}\right) \ldots \phi_{2 n}=\left(0, \varphi_{n}\right)\right\}$, then $u_{h}=\left(u_{h}^{x}, u_{h}^{y}\right) \in V_{h}$ can be gives by the relation $u_{h}=u_{1}^{x} \phi_{1}+\ldots+u_{n}^{x} \phi_{n}+u_{1}^{y} \phi_{n+1}+\ldots+u_{n}^{y} \phi_{2 n}$. For a given triangle $\mathrm{K}$, the displacement field $u_{h}$ and the divergence $\psi_{h}$ are approximated by linear combinations of the basis functions in the form $u_{h}^{x}=\sum_{i=1}^{3} u_{i}^{x} \varphi_{i}+u_{b} \varphi_{b}, u_{h}^{y}=$ $\sum_{i=1}^{3} u_{i}^{y} \varphi_{i}+u_{b} \varphi_{b}, \psi_{h}=\sum_{i=1}^{3} \psi_{i} \varphi_{i}$. The linear system (4.4), attached to the discrete system (4.2) is evaluated over each triangle $\mathrm{K}$ to obtain the element of the local matrices and The global matrices are denoted by uppercase letters, and are given by direct-summing. The matrices elements over the domain 
$\Omega$ are given by

$$
\begin{array}{r}
a_{i j}=\int_{K} \mu \nabla \varphi_{i} . \nabla \varphi_{j} d K, \quad A=\sum_{K \in T_{h}} a_{i j} \\
b_{i j}^{x}=\int_{K}(\lambda+\mu) \frac{\partial \varphi_{i}}{\partial x} \varphi_{j} d K, \quad B^{x}=\sum_{K \in T_{h}} b_{i j}^{x} \\
b_{i j}^{y}=\int_{K}(\lambda+\mu) \frac{\partial \varphi_{i}}{\partial y} \varphi_{j} d K, \quad B^{y}=\sum_{K \in T_{h}} b_{i j}^{y} \\
b_{N_{i j}}^{x}=b_{i j}^{x}-\int_{E \cap K \subset \Gamma_{N, h}} \mu \varphi_{i} n_{i j} \varphi_{j} d E, B_{N}^{x}=\sum_{K \in T_{h}} b_{N_{i j}}^{x} \\
b_{N_{i j}}^{y}=b_{i j}^{y}-\int_{E \cap K \subset \Gamma_{N, h}} \mu \varphi_{i} n_{i j} \varphi_{j} d E, B_{N}^{y}=\sum_{K \in T_{h}} b_{N_{i j}}^{y} \\
d_{i j}=\int_{K}(\lambda+\mu) \varphi_{i} \varphi_{j} d K, \quad D=\sum_{K \in T_{h}} d_{i j} \\
l_{i}^{x}=\int_{K} f_{1} \varphi_{i} d K+\int_{E \cap K \subset \Gamma_{N, h}} g_{1} \varphi_{i} d E, \quad L^{x}=\sum_{K \in T_{h}} l_{i}^{x} \\
l_{i}^{y}=\int_{K} f_{2} \varphi_{i} d K+\int_{E \cap K \subset \Gamma_{N, h}} g_{2} \varphi_{i}, \quad L^{y}=\sum_{K \in T_{h}} l_{i}^{y} d E
\end{array}
$$

knowing that $f=\left(f_{1}, f_{2}\right), g=\left(g_{1}, g_{2}\right), n_{i j}=0,1$ or -1 , we have element matrices.

In this section, we Calculate elementary matrices in (4.5), In order to carry them out,the calculations which have already been made in the article [2] will be based.

For a triangle $\mathrm{K}$ let $\left(x_{i} ; y_{i}\right)_{i=1,2,3}$ be the vertices and the basis functions are defined by $\varphi_{1}(x, y)=1-x-y, \varphi_{2}(x, y)=x, \varphi_{3}(x, y)=y, \varphi_{b}=27 \varphi_{1} \varphi_{2} \varphi_{3}$. We need the following notations, $x_{i j}=x_{i}-x_{j}, y_{i j}=y_{i}-y_{j} \quad i, j=1,2,3$ and

$$
x^{(K)}=\left(\begin{array}{l}
x_{32} \\
x_{13} \\
x_{21}
\end{array}\right)=\left(\begin{array}{c}
x_{1}^{(K)} \\
x_{2}^{(K)} \\
x_{3}^{(K)}
\end{array}\right), y^{(K)}=\left(\begin{array}{l}
y_{32} \\
y_{13} \\
y_{21}
\end{array}\right)=\left(\begin{array}{c}
y_{1}^{(K)} \\
y_{2}^{(K)} \\
y_{3}^{(K)}
\end{array}\right)
$$

the nonbubble part of the matrix $\mathrm{A}$ is written like $A^{K}=\left(a_{i j}\right)_{i, j=1,2,3}=\frac{\mu}{4|K|}\left(y^{(K)} y^{(K)_{t}}+x^{(K)} x^{(K) t}\right)$, with $|K|=\frac{x_{3}^{(K)} y_{1}^{(K)}-x_{2}^{(K)} y_{3}^{(K)}}{2}$ the bubble part of A are $A_{b .}=\left(a_{b j}\right)_{j=1,2,3}=\mathbf{0}_{31}$, with $\mathbf{0}_{31}$ is a Zero column vector with 3 elements 
And for the diagonal

$$
\begin{aligned}
a_{b b} & =\frac{81 \mu}{10}|K|\left(\left|\nabla \varphi_{1}\right|^{2}+\left|\nabla \varphi_{2}\right|^{2}+\nabla \varphi_{1} \cdot \nabla \varphi_{2}\right) \\
& =\frac{81 \mu}{40} \frac{1}{|K|}\left(\left(x_{1}^{(K)}\right)^{2}+\left(x_{2}^{(K)}\right)^{2}+\left(y_{1}^{(K)}\right)^{2}+\left(y_{2}^{(K)}\right)^{2}+x_{1}^{(K)} x_{2}^{(K)}+y_{1}^{(K)} y_{2}^{(K)}\right)
\end{aligned}
$$

Since the stiffness matrix $A$ is symmetric,then we have

$$
A=\left(\begin{array}{cc}
A^{K} & A_{b .} \\
A_{b .}^{t} & a_{b b}
\end{array}\right)=\left(\begin{array}{cc}
A^{K} & 0 \\
0 & a_{b b}
\end{array}\right)
$$

the the mass matrix $\mathrm{D}$ is given by for $\mathrm{i}, \mathrm{j}=1,2,3$

$$
D=\left\{\begin{array}{l}
\frac{\mu+\lambda}{6}|K|, i=j \\
\frac{\mu+\lambda}{12}|K| \quad i \neq j
\end{array}\right.
$$

Now we will implement the divergence matrices, the element matrices of nonbubble part of $B_{x}$ and $B_{x}$ are given by

$$
B_{x}^{K}=\frac{1}{6}(\mu+\lambda)\left(\begin{array}{l}
y^{(K)_{t}} \\
y^{(K)_{t}} \\
y^{(K)_{t}}
\end{array}\right) \quad, \quad B_{y}^{K}=\frac{1}{6}(\mu+\lambda)\left(\begin{array}{l}
x^{(K)_{t}} \\
x^{(K)_{t}} \\
x^{(K)_{t}}
\end{array}\right)
$$

the bubble part are given like $B_{x b}=(\mu+\lambda) \frac{9}{40} y^{(K)_{t}}, B_{y b}=(\mu+\lambda) \frac{9}{40} x^{(K)_{t}}$ Finally

$$
B_{x}=\left(\begin{array}{l}
B_{x}^{K} \\
B_{x b}
\end{array}\right) \quad, \quad B_{y}=\left(\begin{array}{c}
B_{y}^{K} \\
B_{y b}
\end{array}\right)
$$

\section{Element right-hand side}

The nonbubble part of the external forces is given by

$$
l_{x}^{K}=\frac{|K|}{3} f_{1 K}\left(\begin{array}{l}
1 \\
1 \\
1
\end{array}\right), l_{y}^{K}=\frac{|K|}{3} f_{2 K}\left(\begin{array}{l}
1 \\
1 \\
1
\end{array}\right)
$$

where

$$
f_{i K}=\frac{\left(f_{i}\left(x_{1}\right)+f_{i}\left(x_{2}\right)+f_{i}\left(x_{3}\right)\right)}{3}, i=1,2
$$

the bubble part of the right-hand side are $l_{x b}=\frac{9}{20}|K| f_{1 K}, l_{y b}=\frac{9}{20}|K|$ $f_{2 K}$, finally we have

$$
l_{x}=\left(\begin{array}{l}
l_{x}^{K} \\
l_{x b}
\end{array}\right), l_{y}=\left(\begin{array}{l}
l_{y}^{K} \\
l_{y b}
\end{array}\right)
$$


Now we will build the matrix of $11 \times 11$ corresponding to the system (4.4)

$$
\left(\begin{array}{ccccc}
A^{k} & 0 & 0 & 0 & B_{N, x}^{t} \\
0 & a_{b b} & 0 & 0 & B_{b N, x}^{t} \\
0 & 0 & A^{k} & 0 & B_{N, y}^{t} \\
0 & 0 & 0 & a_{b b} & B_{b N, y}^{t} \\
B_{x}^{K} & B_{b x} & B_{y}^{K} & B_{b y} & -D
\end{array}\right)\left(\begin{array}{c}
u_{x} \\
u_{b x} \\
u_{y} \\
u_{b y} \\
\psi
\end{array}\right)=\left(\begin{array}{c}
l_{x}^{K} \\
l_{b x} \\
l_{y}^{K} \\
l_{b y} \\
0
\end{array}\right)
$$

We will classify the system (4.13) in such a way that we can describe the bubble part of the unknown $u$ as a function of the nonbubble part of $u$ and $\psi$,in order to be able to eliminate it thereafter

$$
\left(\begin{array}{ccccc}
A^{k} & 0 & 0 & 0 & B_{N, x}^{t} \\
0 & A^{k} & 0 & 0 & B_{N, y}^{t} \\
0 & 0 & a_{b b} & 0 & B_{b N, x}^{t} \\
0 & 0 & 0 & a_{b b} & B_{b N, y}^{t} \\
B_{x}^{K} & B_{y}^{K} & B_{b x} & B_{b y} & -D
\end{array}\right)\left(\begin{array}{c}
u_{x} \\
u_{y} \\
u_{b x} \\
u_{b y} \\
\psi
\end{array}\right)=\left(\begin{array}{c}
l_{x}^{K} \\
l_{y}^{K} \\
l_{b x} \\
l_{b y} \\
0
\end{array}\right)
$$

From line 3 and 4 of the system (4.14),we deduces

$$
\begin{aligned}
& u_{b x}=a_{b b}^{-1}\left(l_{b x}-B_{b N, x}^{t} \psi\right) \\
& u_{b y}=a_{b b}^{-1}\left(l_{b y}-B_{b N, y}^{t} \psi\right)
\end{aligned}
$$

with the elimination of $u_{b x}$ and $u_{b y}$ we find the new linear system with the Matrix in $\mathbb{U}=\left(\begin{array}{l}u_{x} \\ u_{y} \\ \psi\end{array}\right)$

$$
\mathbb{A}=\left(\begin{array}{ccc}
A^{k} & 0 & B_{N, x}^{t} \\
0 & A^{k} & B_{N, y}^{t} \\
B_{x}^{K} & B_{y}^{K} & -B_{b x} B_{b N, x}^{t} a_{b b}^{-1}-B_{b y} B_{b N, y}^{t} a_{b b}^{-1}-D
\end{array}\right)
$$

and the right hand

$$
\mathbb{F}=\left(\begin{array}{c}
l_{x}^{K} \\
l_{y}^{K} \\
-B_{b x} l_{b x} a_{b b}^{-1}-B_{b y} l_{b y} a_{b b}^{-1}
\end{array}\right)
$$

In this paragraph we presented the assembling of the matrix $\mathbb{A}$ and the right hand $\mathbb{F}$, using the matlab code in Article [2], we modified and adapted 
this code to our problem. The main difficulty in solving the linear system $\mathbb{A} \mathbb{U}=\mathbb{F}$ by using Matlab is the The implementation of neumann terms in integrals Constituting the elements of the matrices $B_{N, x}, B_{N, y}, B_{b N, x}, B_{b N, y}$. The Matlab code contains two main routines. One assembles the matrix $\mathbb{A}$, called

Function $\mathrm{A}=$ elastic2dp1bmat2 (p, t, lambda, $\mathrm{mu}, \mathrm{nn}$ ). The other routine assembles the vector $\mathbb{F}$,called Function $\mathrm{b}=$ elastic2dp1brhs2 (p, t, lambda, mu, $\mathrm{f} 1, \mathrm{f} 2$ ).knowing that $\mathrm{p}$ and $\mathrm{t}$ are two arrays that store nodes coordinates and triangle vertices Sequentially, nn, nt and np are the The numbers of neumann nodes,triangle vertices and, nodes coordinates Sequentially

\section{Assembly of the matrix $\mathbb{A}$ : loop over triangles}

function $\mathrm{A}=$ elastic2dp1bmat2(p, t, lambda, mu, nn)

$\mathrm{np}=\operatorname{size}(\mathrm{p}, 1) ; \mathrm{nt}=\operatorname{size}(\mathrm{t}, 1) ; \mathrm{Z}=\operatorname{zeros}(3,3) ;$

$\mathrm{Mel}=(1 / 12)^{*}[2 \quad 1 \quad 1 ; 1 \quad 2 \quad 1 ; 1112]$;

loop over all triangles

if $($ nargin $==5), \mathrm{A}=\operatorname{sparse}\left(3^{*} \mathrm{np}, 3^{*} \mathrm{np}, \mathrm{nn}\right)$;

else, $\mathrm{A}=\operatorname{sparse}\left(3^{*} \mathrm{np}, 3^{*} \mathrm{np}\right)$; end

for $\mathrm{ih}=1: \mathrm{nt}$

it $=\left[\mathrm{t}(\mathrm{ih}, 1: 3), \mathrm{np}+\mathrm{t}(\mathrm{ih}, 1: 3), 2^{*} \mathrm{np}+\mathrm{t}(\mathrm{ih}, 1: 3)\right]$;

triangles area

$x 21=p(t(i h, 2), 1)-p(t(i h, 1), 1) ; y 12=p(t(i h, 1), 2)-p(t(i h, 2), 2) ;$

$x 32=p(t(i h, 3), 1)-p(t(i h, 2), 1) ; y 23=p(t(i h, 2), 2)-p(t(i h, 3), 2)$;

$x 13=p(t(i h, 1), 1)-p(t(i h, 3), 1) ; y 31=p(t(i h, 3), 2)-p(t(i h, 1), 2)$;

tarea $=\left(\mathrm{x} 21 * \mathrm{y} 31-\mathrm{x} 13^{*} \mathrm{y} 12\right) / 2 ;$

$x^{(T)}, y^{(T)}$, and abb

$\mathrm{xt}=[\mathrm{x} 32 ; \mathrm{x} 13 ; \mathrm{x} 21] ; \mathrm{yt}=[\mathrm{y} 23 ; \mathrm{y} 31 ; \mathrm{y} 12] ;$

$a b b=(81 / 40) * m u *\left((y 23)^{2}+(x 32)^{2}+(y 31)^{2}+(x 13)^{2}+y 23 * y 31+x 32 *\right.$ $x 13) /$ tarea ;

element stiffness matrices : A

$A h=m u *(0.25 /$ tarea $) *\left(x t * x t^{\prime}+y t * y t^{\prime}\right) ;$

element divergence/stabilization matrices

$B N x=($ lambda $+m u) *(1 / 6) *\left[y t^{\prime} ; y t^{\prime} ; y t^{\prime}\right] ;$

$B N y=(l a m b d a+m u) *(1 / 6) *\left[x t^{\prime} ; x t^{\prime} ; x t^{\prime}\right] ;$

$B x=(l a m b d a+m u) *(1 / 6) *\left[y t^{\prime} ; y t^{\prime} ; y t^{\prime}\right]$; 
$B y=(l a m b d a+m u) *(1 / 6) *\left[x t^{\prime} ; x t^{\prime} ; x t^{\prime}\right] ;$

$E=-(l a m b d a+m u)^{2} * 81 /(1600 * a b b) *\left(y t * y t^{\prime}+x t * x t^{\prime}\right)-(l a m b d a+m u) *$ tarea $*$ Mel ;

element P1-bubble/P1 matrix and assembly

Ael $=[$ Ah Z BNx';Z Ah BNy'; Bx By E $] ; \mathrm{A}($ it,it $)=\mathrm{A}(\mathrm{it}, \mathrm{it})+$ Ael $;$ end

\section{Assembly of the right-hand side : loop over triangles}

function $\mathrm{b}=$ elastic2dp1brhs2(p, t, lambda, mu, f1, f2)

$\mathrm{np}=\operatorname{size}(\mathrm{p}, 1) \quad ; \mathrm{nt}=\operatorname{size}(\mathrm{t}, 1)$;

loop over all triangles

$\mathrm{b}=\operatorname{zeros}\left(3^{*}\right.$ np, 1$)$;

for ih $=1: \mathrm{nt}$

it $=\left[\mathrm{t}(\mathrm{ih}, 1: 3), \mathrm{np}+\mathrm{t}(\mathrm{ih}, 1: 3), 2^{*} \mathrm{np}+\mathrm{t}(\mathrm{ih}, 1: 3)\right]$;

triangles area

$x 21=p(t(i h, 2), 1)-p(t(i h, 1), 1) ; y 12=p(t(i h, 1), 2)-p(t(i h, 2), 2) ;$

$x 32=p(t(i h, 3), 1)-p(t(i h, 2), 1) ; y 23=p(t(i h, 2), 2)-p(t(i h, 3), 2) ;$

$x 13=p(t(i h, 1), 1)-p(t(i h, 3), 1) ; y 31=p(t(i h, 3), 2)-p(t(i h, 1), 2) ;$

tarea $=\left(\mathrm{x} 21^{*} \mathrm{y} 31-\mathrm{x} 13^{*} \mathrm{y} 12\right) / 2 ;$

(f1, f2) at the center of triangles

$\mathrm{f} 1 \mathrm{t}=(\mathrm{f} 1(\mathrm{t}(\mathrm{ih}, 1))+\mathrm{f} 1(\mathrm{t}(\mathrm{ih}, 2))+\mathrm{f} 1(\mathrm{t}(\mathrm{ih}, 3))) / 3 ;$

$\mathrm{f} 2 \mathrm{t}=(\mathrm{f} 2(\mathrm{t}(\mathrm{ih}, 1))+\mathrm{f} 2(\mathrm{t}(\mathrm{ih}, 2))+\mathrm{f} 2(\mathrm{t}(\mathrm{ih}, 3))) / 3$;

$\left.x^{(} T\right), y^{(T)}$, abb

$\mathrm{xt}=[\mathrm{x} 32 ; \mathrm{x} 13 ; \mathrm{x} 21] ; \mathrm{yt}=[\mathrm{y} 23 ; \mathrm{y} 31 ; \mathrm{y} 12] ;$

$a b b=(81 / 40) * m u *\left((y 23)^{2}+(x 32)^{2}+(y 31)^{2}+(x 13)^{2}+y 23 * y 31+x 32 *\right.$

x13)/tarea ;

element right-hand side

$\mathrm{lxb}=(9 / 20) *$ tarea $*$ f1t $/ \mathrm{abb} ; \mathrm{lyb}=(9 / 20){ }^{*}$ tarea*f2t $/ \mathrm{abb} ;$

$\mathrm{lx}=(1 / 3) * \mathrm{f} 1 \mathrm{t} *$ tarea $* \operatorname{ones}(3,1) ;$

ly $=(1 / 3) *$ f2t $*$ tarea $* \operatorname{ones}(3,1) ;$

$\mathrm{lb}=-($ lambda $+\mathrm{mu}) / \mathrm{abb} *((9 / 40) * 1 \mathrm{xb} * \mathrm{yt}+(9 / 40) * 1 \mathrm{yb} * \mathrm{xt}) ;$

Assembly

$\mathrm{b}(\mathrm{it})=\mathrm{b}(\mathrm{it})+[\mathrm{lx} ; \mathrm{ly} ; \mathrm{lb}]$; end

\section{Inserting the boundary conditions of Dirichlet}

DirichletNodes $=$ unique ( dirichlet $)$; 
$[W, M]=u_{d}($ coordinates $($ DirichletNodes,$:))$;

$\mathrm{R}=\operatorname{sparse}(\operatorname{size}(\mathrm{W}, 1), 3 *$ size (coordinates, 1$))$;

for $\mathrm{k}=0: 1$

for $1=0: 1$

$\mathrm{R}\left(1+1: 2\right.$ :size $(\mathrm{M}, 1), 3^{*}$ DirichletNodes- $\left.1+\mathrm{k}\right)=\operatorname{diag}(\mathrm{M}(1+1: 2:$

size $(\mathrm{M}, 1), 1+\mathrm{k}))$;

end

end

\section{Inserting the boundary conditions of Neumann}

if isempty(neumann)

$\mathrm{n}=(\operatorname{coordinates}($ neumann $(:, 2),:)-\operatorname{coordinates}($ neumann $(:, 1),:)) *[0,-1 ; 1,0]$;

for $\mathrm{j}=1$ : size(neumann, 1 ) ;

$\mathrm{I}=3^{*}$ neumann $(\mathrm{j},[1,1,2,2])-[1,0,1,0]$;

$\mathrm{gm}=\mathrm{g}(\operatorname{sum}(\operatorname{coordinates}(\operatorname{neumann}(\mathrm{j},:),:)) / 2, \mathrm{n}(\mathrm{j},:) / \operatorname{norm}(\mathrm{n}(\mathrm{j},:)))^{\prime}$;

$\mathrm{b}(\mathrm{I})=\mathrm{b}(\mathrm{I})+\operatorname{norm}(\mathrm{n}(\mathrm{j},:)) *[\mathrm{gm} ; \mathrm{gm}] / 2 ;$

end

end

\section{NUMERICAL RESULTS}

This section is the fruit of the study that is done in the previous sections in this paper, with its two sides: mathematics and programming. So there is some numerical results of calculations with mixed finite element P1 bublle-P1 Method that will be presented later. A two-dimensional problem with the elastic square body with a hole $\Omega=[0,2] \times[0,2] \backslash B(0,1)(\mathrm{E}=2900$ and nu $=0.25)$,is stretched at the top $(\mathrm{y}=2)$ with a surface load $g=n$, where $\mathrm{n}$ denotes the outer normal to $\partial \Omega$; the rest of the boundary is traction free [11].By reason of symmetry since the domain is homogeneous,one will present the quarter of the domain that was discretized.The Dirichlet conditions are:h $=0$ on $[1,2] \times 0$ and $0 \times[1,2]$ the forces charges are taking as $\mathrm{f}=0$ for all nodes.

The main aim of this numerical experiment is to test the stability of the divergence of the field of displacement $u_{h}$, and compare it with the numerical 


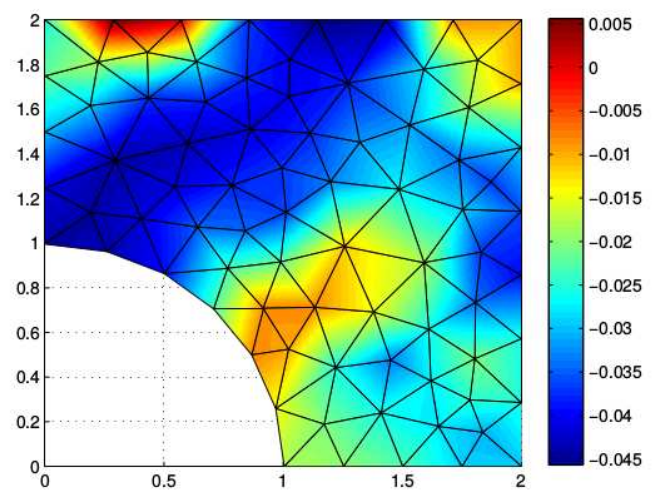

Figure 1: Deformed mesh for membrane with hole $h=0.3$

solution $\psi_{\text {num }}$.In fact,we will calculate the error $e=\left(\frac{\left|d i v u_{h}-\psi_{n u m}\right|}{n p}\right)^{\frac{1}{2}}$ for three meshes,and we will see how this error behaves when the mesh size grows, That is when $h \rightarrow 0$.So from the tabular the error e go to zero when $\mathrm{h}$ is too small.

\begin{tabular}{|r||c|l|l|}
\hline Number of nodes & 80 & 164 & 589 \\
\hline Number of elements & 130 & 287 & 1099 \\
\hline $\mathrm{h}$ & 0.3 & 0.2 & 0.1 \\
\hline error e & 0.2252 & 0.2004 & 0.1699 \\
\hline$\left\|u-u_{h}\right\|_{1, \Omega}$ & 0.0342 & 0.0298 & 0.0146 \\
\hline$\left\|\psi-\psi_{h}\right\|_{0, \Omega}$ & 0.0567 & 0.0346 & 0.0264 \\
\hline
\end{tabular}

The example demonstrates how the mixed finite element P1 bubble-P1 method is more efficient then the ordinary one.Because it allows us to calculate the displacements and their divergences simultaneously,and that it guarantees the stability of these divergences on each node.

\section{ACKNOWLEDGMENTS}

The authors would like to appreciate the referees for giving us the several corrections The authors declares that there is no conflict of interest regarding the publication of this paper. 


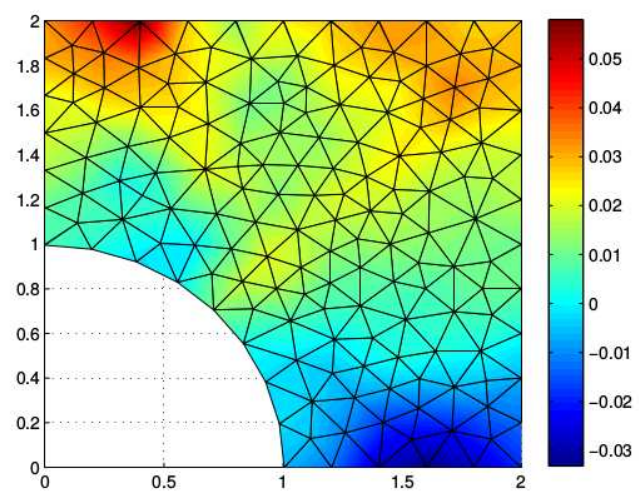

Figure 2: Deformed mesh for membrane with hole $h=0.2$

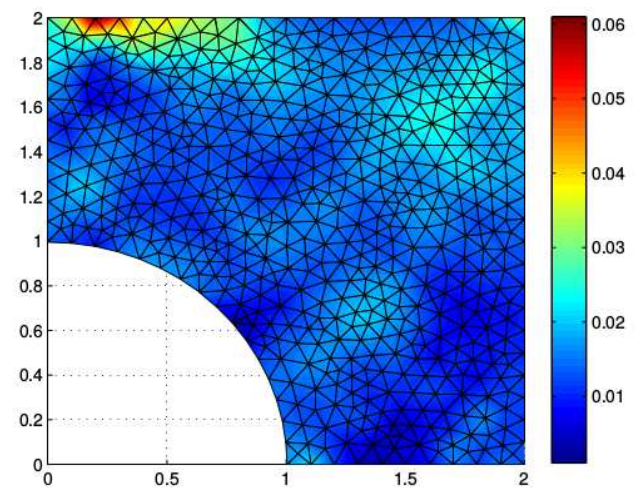

Figure 3: Deformed mesh for membrane with hole $h=0.1$

\section{REFERENCES}

[1] J. Alberty, C. Carstensen, S.A. Funken, R. Klose, Matlab implementation of the finite element method in elasticity, Computing, 69 (2002), 239-263, doi: $10.1007 / \mathrm{s} 00607-002-1459-8$.

[2] Jonas Koko, Vectorized Matlab codes for the Stokes problem with $P^{1}$ bubble $/ P^{1}$ finite element, Universite Blaise Pascal -CNRS UMR 6158 ISIMA, Campus des Cezeaux - BP 10125, 63173, France.

[3] P. Ciarlet, J.R. Jianguo Huang, Jun Zou, Some observation ongeneralized saddle-point problem. 
[4] D.N. Arnold, F. Brezzi, and M. Fortin, A stable finite element for the stokes equations, Calcolo, 21 (1984), 337-344.

[5] K.J. Bathe, Finite Element Procedures, Second Edition, K.J. Bathe, Watertown, MA, 2014.

[6] V. Girault, P.A. Raviart, Finite Element Approximation of the NavierStokes Equations, Springer-Verlag, Berlin Heiderlberg New York, 1981.

[7] Alexandre Ern, Aide-Memoire Elements Finis, Dunod, Paris, 2005.

[8] F. Brezzi and M. Fortin, Mixed and Hybrid Element Methods, SpringerVerlag, New York, 1991.

[9] R.B. Kellogg and B. Liu, A finite element method for compressible Stokes equations, SIAM J. Numer. Anal., 33 (1996), 780-788.

[10] D. Yang, Iterative schemes for mixed finite element methods with applications to elasticity and compressible flow problems, Numer. Math., 93 (2002), 177-200.

[11] P. Wriggers, et al. Benchmark perforated tension strip. Communication in talk at Enumath Conference in Heidelberg, 1997 (see also http://www.ibnm.unihannover.de/Forschung/Paketantrag/Benchmarks/benchmark.html) 